\title{
Using linkage maps to correct and scaffold de novo genome assemblies: methods, challenges, and computational tools
}

OPEN ACCESS

Edited by:

Max A. Alekseyev,

George Washington University, USA

Reviewed by:

Cuncong Zhong,

J. Craig Venter Institute, USA

Martin Mascher

Institut für Pflanzengenetik und Kulturpflanzenforschung Gatersleben,

Germany

Matthew Hahn Indiana University, USA Christopher R. Smith,

Earlham College, USA

*Correspondence: Janna L. Fierst, Department of Biological Sciences, University of Alabama, Box 870344

Tuscaloosa, AL 35847, USA jlfierst@ua.edu

Specialty section: This article was submitted to Bioinformatics and Computational

Biology,

a section of the journal

Frontiers in Genetics

Received: 08 April 2015

Accepted: 08 June 2015

Published: 19 June 2015

Citation:

Fierst JL (2015) Using linkage maps to correct and scaffold de novo genome assemblies: methods, challenges, and computational tools. Front. Genet. 6:220

doi: 10.3389/fgene.2015.00220

\author{
Janna L. Fierst * \\ Department of Biological Sciences, University of Alabama, Tuscaloosa, AL, USA
}

Modern high-throughput DNA sequencing has made it possible to inexpensively produce genome sequences, but in practice many of these draft genomes are fragmented and incomplete. Genetic linkage maps based on recombination rates between physical markers have been used in biology for over 100 years and a linkage map, when paired with a de novo sequencing project, can resolve mis-assemblies and anchor chromosome-scale sequences. Here, I summarize the methodology behind integrating de novo assemblies and genetic linkage maps, outline the current challenges, review the available software tools, and discuss new mapping technologies.

Keywords: next-generation sequencing, draft genome, scaffolds, physical mapping, optical mapping

\section{Introduction}

De novo genome sequences are fueling a scientific revolution. Biologists are in a position to answer questions that were unimaginable 30 years ago, and new technologies and resources are generating new questions. However, many of these draft genomes contain thousands of individual sequences with no information on how these pieces are assembled into chromosomes. This is problematic both for molecular and developmental studies as individual genes may end up fractured and incorrectly annotated (Baker, 2012; Denton et al., 2014) and for evolutionary studies as fragmented sequences lack the genomic context that is necessary to analyze comparative patterns. For example, the analysis of 12 genomes from closely related Drosophila species found increased codon bias and rates of adaptive substitution in genes residing on the $\mathrm{X}$ chromosome (Drosophila 12 Genomes Consortium, 2007). Relying solely on DNA sequencing means there is no way to identify mistakes in the assembled genome sequence and without a high-quality way to evaluate, correct and anchor next-generation assemblies, they are of limited use.

De novo sequencing projects can be successfully paired with a linkage map to address these shortcomings (Semagn et al., 2006; Lewin et al., 2009). Millions of genetic markers can be readily produced with high-throughput sequencing (Baird et al., 2008; Elshire et al., 2011; Heffelfinger et al., 2014), although these large-scale datasets present significant statistical and computational challenges. Genetic linkage maps have been used to refine de novo assemblies in organisms ranging from the commercial potato (Xu et al., 2011) to the collared flycatcher bird (Kawakami et al., 2014). There are currently few resources on integrating de novo assemblies with linkage maps, particularly for researchers without extensive statistical or computational backgrounds. This article is meant to be a primer for a wide range of biologists interested in using these methods. Below, I outline the scientific problems involved in generating de novo assemblies and linkage maps, explain how the 
two can be integrated, summarize existing computational tools, and describe new technologies for generating physical maps.

\section{Next Generation Genome Assembly}

De novo genome assembly works by extracting and sequencing small segments of DNA molecules, and piecing these segments back together into contigs, contiguous sequences in which every nucleotide is known (i.e., A, C, G, or T), and scaffolds, sequences that contain regions with unknown nucleotides (i.e., $\mathrm{N})$. Next-generation sequencing-by-synthesis has shrunk the price of a million bases of sequenced DNA from $\$ 2400$ (with Sanger chain-termination sequencing; Sanger and Coulson, 1975; Sanger et al., 1977) to $<\$ 0.25$ (Liu et al., 2012). The reduced cost makes it feasible for individual investigators to undertake genome sequencing projects, but it carries decreases in read length and accuracy. Sanger sequencing produces $400-900 \mathrm{bp}$ sequencing reads with a per-base accuracy of $99.9 \%$ compared to 50-300 bp sequencing-by-synthesis reads (although long reads are possible) (Petterson et al., 2009; Quail et al., 2012). Nextgeneration sequencing has an average per-base accuracy of $99 \%$ but this decreases systematically with high and low GC bias (Dohm et al., 2008) and results in reduced sequencing of these regions (Kozarewa et al., 2009; Chen et al., 2013).

Inserting 30-350 kbp lengths of DNA into plasmids to create bacterial artificial chromosomes (BACs) (O'Connor et al., 1989; Shizuy et al., 1992), cosmids (Collins and Hohn, 1978), and fosmids (Kim et al., 1992) reduces the complexity of wholegenome assembly by effectively breaking the problem down into smaller segments. These genome segments are sequenced and assembled individually but the process is time-consuming and expensive. Sanger sequencing of plasmid clones at $10 \times$ depth (where each nucleotide is sequenced, on average, 10 times) can adequately represent the $3 \mathrm{~Gb},>50 \%$ repetitive human genome (Green, 1997; Weber and Myers, 1997). With fewer reads and longer lengths overlap/layout/consensus (OLC) assembly, in which all sequencing reads are compared pairwise and assembled based on overlap, is feasible (Myers et al., 2000; Batzoglou et al., 2002). Short read lengths require $>100 \times$ depth and assembling these large, complex datasets requires sophisticated algorithms like de Bruijn graphs (Pevzner et al., 2001), in which sequencing reads are broken down into short segments of length $k$ and these $k$-mers connected in large graphs (Pevzner et al., 2001; Zerbino and Birney, 2010).

Even with sophisticated assembly algorithms, short sequencing reads alone can not generate the information that is needed to discriminate genomic repeats and duplications (Pop and Salzberg, 2008; Alkan et al., 2011) or ancestral polyploidy (The International Wheat Genome Sequencing Consortium, 2014; Chapman et al., 2015). Many current genome sequencing projects rely on mate-pair libraries for long-range sampling throughout the genome (for a review of sequencing strategies, see Ekblom and Wolf, 2014). These specialized libraries select 1-15 kb segments of DNA for circular ligation and extract the ligated ends for traditional short-read sequencing. Assembly algorithms build contiguous sequences from short sequencing reads and use the long-range information provided by mate-pairs to construct large scaffolds (Gnerre et al., 2011). The resulting sequences have high per-base accuracy in gene-rich regions of the genome but do not approximate finished genome sequences (Alkan et al., 2011).

Whole genome shotgun (WGS) assemblies have always suffered from the same limitations, and short read lengths have amplified these problems (Earl et al., 2011; Bradnam et al., 2013; Figure 1A). First, WGS assemblies are inherently fragmented. Eukaryotic genomes contain, at a minimum, millions of nucleotides and "long" contiguous sequences do not approach chromosome-scale. Next-generation assemblies contain many small fragments (on the order of 1000's of nucleotides), and these provide little genetic information. Second, repeat elements are difficult to assemble and can result in mis-joins. Third, diploid individuals, even after extensive inbreeding, will often have residual heterozygosity (Price et al., 2012). These sequences assemble poorly and sometimes occur as duplicated fragments in the assembled sequence. The program REAPR (Hunt et al., 2013) evaluates assembly quality by re-aligning the DNA sequences to the assembled genome but beyond this assessing quality must be done through contig/scaffold length statistics and heuristics combining protein-coding gene annotations with comparative expectations from related species with high-quality assembled sequences (Ekblom and Wolf, 2014). Without a secondary source of information, there is no rigorous way to identify errors.

Longer sequencing reads can build larger contiguous sequences and facilitate higher quality de novo assemblies (Alkan et al., 2011) but each of the new platforms has critical shortcomings. Illumina TruSeq synthetic long reads range up to $18,500 \mathrm{bp}$ (McCoy et al., 2014) but rely on parallel library preparation coupled with traditional short read sequencing and bias against assembly of repeats and duplications (Koren and Phillippy, 2015). The Oxford Nanopore MinION passes a single strand of DNA through a protein nanopore (Schneider and Dekker, 2012) and produces reads $>20,000$ bp but the per-base accuracy is just $70-80 \%$ (Quick et al., 2014). Pacific Biosciences single molecule real time (SMRT) sequencing (Eid et al., 2008) produces reads with a median length of $3122 \mathrm{bp}$ but the per-base accuracy is $87 \%$ (Koren et al., 2013). Pacific Biosciences long reads can reduce assembly fragmentation when paired with short DNA sequencing reads and this strategy was used to assemble a 128 contig genome sequence for Drosophila melanogaster (Landolin et al., 2014). Coupling long-read sequencing with short-read sequencing and assembly will require the development of sophisticated error-correction and assembly algorithms (Koren and Phillippy, 2015).

Moving beyond fragmented genome assemblies requires a linkage map (Lewin et al., 2009; Mascher and Stein, 2014). A high-density linkage map can anchor de novo sequences and orient and order small fragments into chromosomescale sequences (Figure 1B). Inconsistency between markers in the map and markers in the assembled sequences can indicate incorrectly assembled sequences and residual heterozygosity. These can then be resolved to produce a high-quality reference draft genome. For example, the Potato Genome Sequencing Consortium assembled a $727 \mathrm{Mb}$ genome sequence through deep short-read sequencing on Sanger, 
A
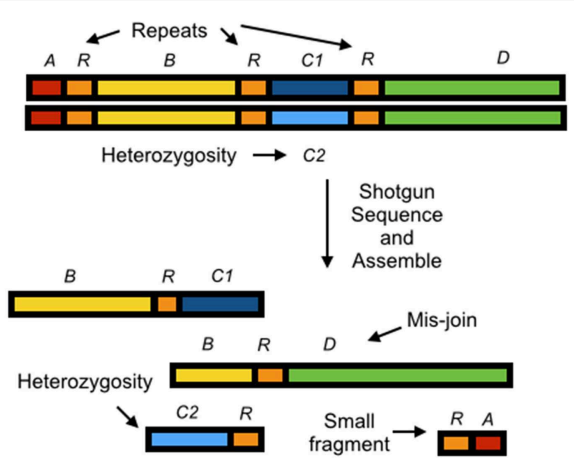

C

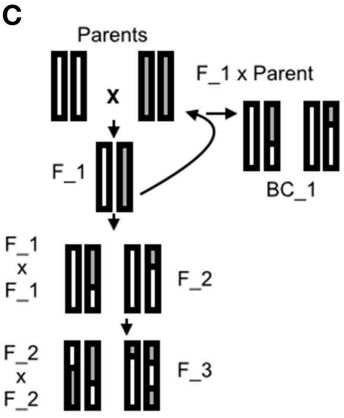

D Marker

M M M M M M $\begin{array}{llllll}1 & 2 & 3 & 4 & 5 & 6\end{array}$

$1 A A B A B A$

$2 A A B B A A$

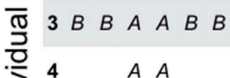

드 5 A $A$ A $B$ B $B \quad B \quad B$

6 A $B$ A $A B$

$\begin{array}{llllll}7 & B & B & B & A\end{array}$

8 B $B$ A $A$ A $B$

FIGURE 1 | (A) In whole genome assembly errors result from residual alleles which appear as discrete sequences in the reference, and mis-joins. Small fragments have no genomic context and contribute little information. (B) Using a genetic linkage map to anchor a de novo assembly resolves error in the reference sequence by giving small sequences genomic context, resolving allelism, and identifying mis-joins. Chromosome-scale assemblies can be constructed by ordering and orienting sequences with the linkage

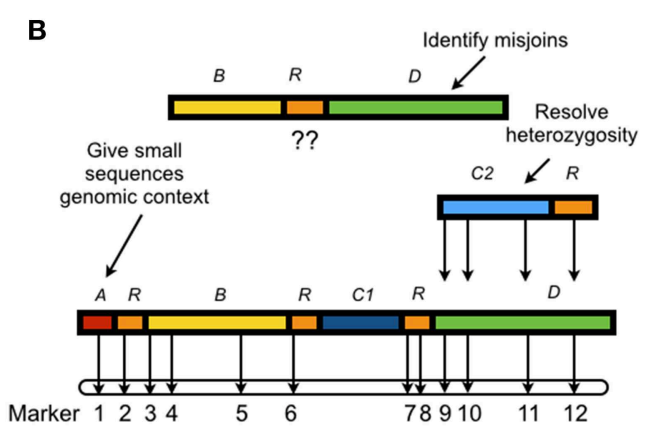

E

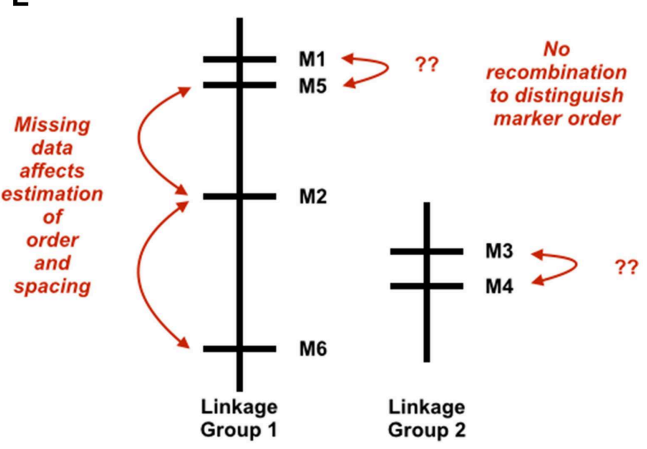

map. (C) A genetic linkage map can be estimated from a parental cross resulting in an F2, F3, or Backcross (here, BC1) population. Estimating a genetic linkage map requires (D) genotyping individuals at discrete markers (here, six markers across eight individuals with missing data); and (E) grouping markers into linkage groups; and ordering and spacing markers within linkage groups. Estimating order and spacing is difficult due to missing data and little recombination between adjacent markers.
Illumina, and Roche 454 platforms (Xu et al., 2011). This deep sequencing resulted in an assembled sequence $90 \%$ of the estimated genome size and spread across 443 superscaffolds, an impressive but complex and fragmented assembly. Construction of a genetic linkage map yielded 12 linkage groups and $86 \%$ of the assembled genome was anchored to these 12 chromosomes.

\section{Genetic Linkage Maps}

The basic mathematical problem of genetic mapping is: given a set of associations between markers, what is the most likely physical arrangement of these markers on chromosomes? In the early days of mapping these were visible markers like eye color in Drosophila (Sturtevant, 1913a,b), at the end of the 20th century these became DNA markers like Restriction Fragment Length Polymorphisms (RFLPs) (Lander and Botstein, 1989), and more recently these have become Single Nucleotide Polymorphism (SNP) markers generated through high-throughput DNA sequencing (for example, Baird et al., 2008; Elshire et al., 2011; Heffelfinger et al., 2014). Constructing a linkage map proceeds in two steps. First, a mapping population must be established to generate recombination and genetic differences between related individuals (Figure 1C). Second, map estimation proceeds by genotyping individuals at different markers (Figure 1D), grouping markers into linkage groups (putative chromosomes), ordering the markers within a group in linear sequence, and spacing the markers according to estimated distances along the chromosome (Figure 1E). Missing data and incorrect marker typing have a large effect on map estimation and infrequent recombination between adjacent markers makes it difficult to order and space markers. These limitations mean that linkage maps are accurate at a large scale but lack fine-scale resolution.

\section{Current Challenges in Using Linkage Maps with De Novo Assemblies}

\section{Establishing a Mapping Population}

Increasing the number of recombination events increases the resolution of the genetic map. This can be achieved by genotyping a very large mapping population but this may be difficult or prohibitively expensive for many organisms. For example, van Oers et al. (2014) constructed a genetic map for the great tit Parus major by SNP genotyping over 2000 individuals created from an F_2 cross. For organisms like maize that can be easily 
bred Recombinant Inbred Lines (RILs) can be established from parental crosses and used for genetic mapping (Burr et al., 1988; Burr and Burr, 1991). However, the necessary time and investment can be prohibitive for long-lived organisms or those that are difficult to breed or grow in the lab. Genetic linkage maps may be estimated from F1 populations (for example, Eucalyptus grandis Bartholome et al., 2015) but this requires different algorithms and is not supported by all map estimation software.

\section{Next-generation Sequencing Markers}

The methodology for estimating linkage maps was originally developed for small-scale data, on the order of hundreds of markers, instead of the millions of genetic markers that are readily produced with high-throughput sequencing (Cheema and Dicks, 2009). The number of possible different orders of genetic markers scales exponentially with the number of markers, and is a major limiting factor in constructing a linkage map. For example, 5 genetic markers in the same linkage group can be ordered in 60 different ways ( $\frac{1}{2} m !$, where $m$ is the number of markers) while 10 genetic markers can be ordered in 1.8 million different ways. The necessary marker density depends on assembly contiguity, and fragmented genome sequences require dense maps for anchoring and orientation. Grouping, ordering and spacing dense marker sets is a central computational challenge and efficient algorithms are still under development (Wu et al., 2008; Strnadova et al., 2014).

Incorrect genotypes and missing data can have a large effect on genetic map estimation, and these problems are magnified by noisy high-throughput SNP genetic markers. Two or more SNPs may be artificially collapsed to a single marker because of sequence similarity in repeats, low-complexity regions, and paralogous genes. Biased sequencing errors may cause one locus to be split into two and uneven sequencing coverage may result from GC bias in polymerase chain reaction (PCR) and sequencing. Sequencing coverage can be uneven across both genomic regions and alleles at one locus due to local GC content. This can result in different data missing from each individual and a negative relationship between sample sizes for markers and individuals.

\section{No Existing Software Tools to Automate the Process}

Map-assembly integration can proceed in two different ways. For sequence-based mapping genetic markers are aligned to the draft assembly and these markers are used to construct a map, while for array-based mapping the map is constructed first and genetic markers aligned second. For both procedures multiply-mapped markers and loci must be excluded from the final map. However, there is no software to perform either of these processes and it requires custom scripting. Misassembled scaffolds can be identified through marker segregation patterns, but in practice identifying and correcting these errors must be done manually. For a typical de novo assembly containing thousands of scaffolds and thousands of genetic markers, this quickly becomes time-consuming and subject to error.

\section{Tools for Estimating Genetic Linkage Maps}

In Table 1 I summarize software packages for estimating genetic linkage maps that have been used to generate a published map and updated since 2008. Currently there is no single software package that integrates completely with de novo assembly, and efficient methods and algorithms are spread across different packages. My goal is to describe the benefits and limitations of each package so biologists can choose which to implement in their own work. For a review of older software, see Cheema and Dicks (2009).

There are several different algorithms for estimating genetic maps (for detailed descriptions of mapping algorithms and performance comparisons see Mollinari et al., 2009; Wu et al., 2011) but these can be generally divided into those that couple iterative marker ordering with probability-based sampling and those that implement graph-based algorithms based on the traveling salesman problem (TSP) (Wu et al., 2008). Under the latter different loci are nodes in a graph and the TSP attempts to connect loci by visiting each node once and only once. The nodes are connected by edges, and the shortest path through the graph is the minimum spanning tree (MST) which approximates the linkage structure underlying the loci. Graph-based algorithms are capable of ordering $>10,000$ loci (Wu et al., 2008; Rastas et al., 2013). In comparison, marker ordering and sampling algorithms are typically capable of ordering $<3000$ markers (Margarido et al., 2007; Wu et al., 2008; Cheema and Dicks, 2009; van Ooijen, 2011).

\section{Developing Integrated Approaches}

Independent genome assembly and map construction can be prohibitively expensive or fail to provide a high-quality assembled sequence for organisms with large, complex, repeatheavy, polyploid or highly heterozygous genomes. Three published methods (Mascher et al., 2013; Hahn et al., 2014; Nossa et al., 2014) integrate whole genome sequencing with linkage map construction in genome assembly, variant calling, map estimation, and map-assisted assembly to produce assembled genome sequences. PopSeq (Mascher et al., 2013) was used to order $927 \mathrm{Mb}$ of the complex, $5.1 \mathrm{~Gb}$ barley genome sequence which is composed of $>80 \%$ repeats. Recombinant Population Genome Construction (RPGC) was used in a simulated assembly of the $100 \mathrm{Mb}$ genome of the self-fertile hermaphrodite Caenorhabditis elegans and produced an assembled genome spread across just 88 scaffolds (Hahn et al., 2014). For a review of these methods, see Mascher and Stein (2014). Nossa et al. (2014) combined de novo assembly with linkage mapping to study the organization of the $2.7 \mathrm{~Gb}$ genome of the Atlantic horseshoe crab and uncover an ancestral genome duplication.

Genetic linkage maps and de novo assemblies have two, complementary scales. Linkage maps are accurate at a large, chromosomal scale, but fine scale marker ordering and spacing are inexact due to infrequent recombination between adjacent markers. In contrast, de novo assemblies are accurate at a fine scale (100-1000's of nucleotides) but can not be used 
TABLE 1 | Software packages for estimating genetic linkage maps.

\begin{tabular}{|c|c|c|}
\hline Package name & Strengths & Limitations \\
\hline $\begin{array}{l}\text { R/qtl } \\
\text { (Broman et al., 2003) }\end{array}$ & $\begin{array}{l}\text { Written in R (user-friendly); High functionality; Integrated graphics; } \\
\text { Transparent, open-source implementation; Supported and under } \\
\text { current development }\end{array}$ & $\begin{array}{l}\text { Difficulty handling >1000 markers; No methods to address } \\
\text { bias in high-throughput DNA sequence markers }\end{array}$ \\
\hline $\begin{array}{l}\text { JoinMap } \\
\text { (Stam, 1993; Jansen et al., } \\
\text { 2001; van Ooijen, 2011) }\end{array}$ & $\begin{array}{l}\text { User-friendly Graphical User Interface (GUI); Efficient algorithms for } \\
\text { grouping and ordering <3000 markers }\end{array}$ & $\begin{array}{l}\text { Only available commercially; Not open-source; Difficulty } \\
\text { handling > } 3000 \text { markers; No methods to address bias in } \\
\text { high-throughput DNA sequence markers }\end{array}$ \\
\hline $\begin{array}{l}\text { OneMap } \\
\text { (Margarido et al., 2007) }\end{array}$ & $\begin{array}{l}\text { F1 crosses; Written in R; Integrates with R/qtl's functionality and } \\
\text { graphics; Transparent, open-source implementation; Robust to } \\
\text { genotyping errors and missing data }\end{array}$ & $\begin{array}{l}\text { Difficulty handling >1000 markers; No methods to address } \\
\text { bias in high-throughput DNA sequence markers }\end{array}$ \\
\hline $\begin{array}{l}\text { MSTMap } \\
\text { (Wu et al., 2008) }\end{array}$ & $\begin{array}{l}\text { Efficient algorithms for linkage grouping and marker ordering; Can } \\
\text { handle }>10,000 \text { markers }\end{array}$ & $\begin{array}{l}\text { Can not handle F1 crosses; Little documentation; Currently } \\
\text { unsupported and may not be under further development; } \\
\text { No methods to address bias in high-throughput DNA } \\
\text { sequence markers }\end{array}$ \\
\hline $\begin{array}{l}\text { Lep-MAP } \\
\text { (Rastas et al., 2013) }\end{array}$ & $\begin{array}{l}\text { F1 crosses; Can handle }>10,000 \text { markers; Specialized module utilizes } \\
\text { scaffold location of genetic markers in assigning linkage groups }\end{array}$ & $\begin{array}{l}\text { Assumes no recombination in one parent (specialized } \\
\text { Lepidopteran mating system; Suomalainen et al., 1973) }\end{array}$ \\
\hline $\begin{array}{l}\text { HighMap } \\
\text { (Liu et al., 2014) }\end{array}$ & $\begin{array}{l}\text { Can handle >1000 markers; Utilizes high-throughput sequencing errors } \\
\text { in correcting genotyping errors and imputing missing data; Graphics } \\
\text { and evaluation functions }\end{array}$ & Recently published and has not been widely tested \\
\hline
\end{tabular}

to accurately reconstruct chromosome-scale relationships. An integrated approach to de novo genome assembly and genetic linkage mapping could utilize the information in each to build a high-quality reference sequence. These methods are just now beginning to appear in computational tools (Liu et al., 2014). For example, LepMap (Rastas et al., 2013) reduces the complexity of linkage group formation with a specialized module that utilizes the scaffold location of genetic markers.

\section{Physical Genome Maps}

There are several molecular techniques that can generate physical genome maps. Until recently these were prohibitively expensive or difficult to implement but breakthroughs in technology are lowering prices and putting physical maps within reach.

Optical mapping generates ordered, high-resolution maps of restriction sites across single DNA molecules (Schwartz et al., 1993) and can produce high-quality, chromosome-scale physical maps. Optical mapping works by immobilizing single molecules of DNA on a slide, digesting the molecules with restriction enzymes, visualizing the fragments with fluorescence microscopy, and sizing the fragments. The fragments are then pieced together to produce a physical map of the genome with restriction site markers. Optical mapping technology was developed over 20 years ago but its high cost has been prohibitive for most genome projects. Currently, optical maps must still be paired with a high-quality de novo assembly but developing nanotechnologies and single molecule sequencing are pushing optical maps to the forefront of genome technology (Levy-Sakin and Ebenstein, 2013). For example, BioNano Genomics Irys System has reduced the price of optical mapping by an order of magnitude and is a feasible platform for studying structural variation in a human genome (Cao et al., 2014).
$\mathrm{Hi}-\mathrm{C}$ is a molecular technique that cross-links chromatin segments in close physical proximity and quantifies these interactions with high-throughput sequencing (LiebermanAiden et al., 2009). The frequencies with which two regions of chromatin interact generates a distribution indicative of the genomic distance between the loci and sufficient for ordering and orienting an assembled genome sequence (Kaplan and Dekker, 2013). The program LACHESIS (Burton et al., 2013) both constructs the frequency-based physical map and aligns scaffolds to the map. Hi-C requires a difficult molecular protocol (de Wit and de Laat, 2012) and has not been widely adopted for genome assembly although it is currently under commercial development and was used to construct genome sequences for a human and the American alligator (Putnam et al., 2015) and Arabidopsis thaliana (Xie et al., 2015).

Contiguity preserving transposase sequencing (CPT-seq) (Adey et al., 2014) capitalizes on the unique properties of tagmentation, a recently developed method for both fragmenting DNA and appending sequencing adaptors (Adey et al., 2010). Tagmentation fragments DNA with a Tn 5 transposase that binds tightly to target DNA. High molecular weight segments of DNA are extracted and the resulting segments, analogous to a pool of fosmid clones, are sequenced to obtain a phased haplotype (Amini et al., 2014). Combining these phased haplotype segments with an initial genome assembly facilitates the construction of large scaffolds (Adey et al., 2014).

\section{Conclusions}

Coupling de novo assembly with linkage mapping is a powerful way to produce a high-quality reference genome. Map estimation was originally developed as a genetic tool over 100 years ago (Sturtevant, 1913a,b) while assembly-specific algorithms and 
tools are still developing. Linkage maps have proven useful in many different genome assembly projects, and over the next few years assembly-specific algorithms and tools will continue to appear. Physical maps generated with emerging technologies are now becoming feasible for genome sequencing projects.

Dense linkage maps can both orient and order assembled sequences and identify the genetic basis of phenotypic traits. Linkage maps are therefore one of the most important tools we have in genetics. Establishing a mapping population takes time, and undertaking a mapping project is a significant

\section{References}

Adey, A., Kitzman, J. O., Burton, J. N., Daza, R., Kumar, A., Christiansen, L., et al. (2014). In vitro, long-range sequence information for de novo genome assembly via transposase contiguity. Genome Res. 24, 2041-2049. doi: $10.1101 /$ gr. 178319.114

Adey, A., Morrison, H. G., Asan, Xun, X., Kitzman, J. O., Turner, E. H., et al. (2010). Rapid, low-input, low-bias construction of shotgun fragment libraries by high-density in vitro transposition. Genome Biol. 11:R119. doi: 10.1186/gb2010-11-12-r119

Alkan, C., Sajjadian, S., and Eichler, E. E. (2011). Limitations of next-generation genome sequence assembly. Nat. Methods 8, 61-65. doi: 10.1038/nmeth.1527

Amini, S., Pushkarev, D., Christiansen, L., Kostem, E., Royce, T., Turk, C., et al. (2014). Haplotype-resolved whole-genome sequencing by contiguitypreserving transposition and combinatorial indexing. Nat. Genet. 46, 13431349. doi: 10.1038/ng.3119

Baird, N. A., Etter, P. D., Atwood, T. S., Currey, M. C., Shiver, A. L., Lewis, Z. A., et al. (2008). Rapid SNP discovery and genetic mapping using sequenced RAD markers. PLoS ONE 3:e3376. doi: 10.1371/journal.pone.0003376

Baker, M. (2012). De novo genome assembly: what every biologist should know. Nat. Methods 9, 333-337. doi: 10.1038/nmeth.1935

Bartholome, J., Mandrou, E., Mabiala, A., Jenkins, J., Nabihoudine, I., Klopp, C., et al. (2015). High-resolution genetic maps of Eucalyptus improve Eucalyptus grandis genome assembly. New Phytol. 206, 1283-1296. doi: 10.1111/nph.13150

Batzoglou, S., Jaffe, D. B., Stanley, K., Butler, J., Gnerre, S., Mauceli, E., et al. (2002). ARACHE: a whole-genome shotgun assembler. Genome Res. 12, 177-189. doi: 10.1101/gr.208902

Bradnam, K. R., Fass, J. N., Alexandrov, A., Baranay, P., Bechner, M., Birol, I., et al. (2013). Assemblathon 2: evaluating de novo methods of genome assembly in three vertebrate species. Gigascience 2:10. doi: 10.1186/2047-217X-2-10

Broman, K. W., Wu, H., Sen, S., and Churchill, G. A. (2003). R/qtl: QTL mapping in experimental crosses. Bioinformatics 19, 889-890. doi: 10.1093/bioinformatics/btg112

Burr, B., and Burr, F. A. (1991). Recombinant inbreds for molecular mapping in maize. Trends Genet. 7, 55-60.

Burr, B., Burr, F. A., Thompson, K. H., Albertson, M. C., and Stuber, C. W. (1988). Gene mapping with recombinant inbreds in maize. Genetics 118, $519-526$.

Burton, J. N., Adey, A., Patwardhan, R. P., Qiu, R., Kitzman, J. O., and Shendure, J. (2013). Chromosome-scale scaffolding of de novo genome assemblies based on chromatin interactions. Nat. Biotechnol. 31, 1119-1125. doi: 10.1038/ nbt. 2727

Cao, H., Hastie, A. R., Cao, D., Lam, E. T., Sun, Y., Huang, H., et al. (2014). Rapid detection of structural variation in a human genome using nanochannelbased genome mapping technology. Gigascience 3:34. doi: 10.1186/2047217X-3-34

Chapman, J. A., Mascher, M., Buluc, A., Barry, K., Georganas, E., Session, A., et al. (2015). A whole-genome shotgun approach for assembling and anchoring the hexaploid bread wheat genome. Genome Biol. 16, 26. doi: 10.1186/s13059-0150582-8

Cheema, J., and Dicks, J. (2009). Computational approaches and software tools for genetic linkage map estimation in plants. Brief. Bioinformatics 10, 595-608. doi: 10.1093/bib/bbp045 investment of resources. However, linkage maps provide highquality sequences that can not result from de novo assembly alone and every genome project that can reasonably be coupled with a linkage map, should be coupled with a linkage map.

\section{Acknowledgments}

Financial support provided by a grant from the National Institutes of Health (GM096008). Four reviewers provided helpful suggestions that greatly improved the manuscript.

Chen, Y., Liu, T., Yu, C., Chiang, T., and Hwang, C. (2013). Effects of GC bias in next-generation-sequencing data on de novo genome assembly. PLoS ONE 8:e62856. doi: 10.1371/journal.pone.0062856

Collins, J., and Hohn, B. (1978). Cosmids: a type of plasmid gene-cloning vector that is packageable in vitro in bacteriophage lambda heads, Proc. Natl. Acad. Sci. U.S.A. 75, 4242-4246.

de Wit, E., and de Laat, W. (2012). A decade of 3C technologies: insights into nuclear organization. Genes Dev. 26, 11-24. doi: 10.1101/gad.179804.111

Denton, J. F., Lugo-Martinez, J., Tucker, A. E., Schrider, D. R., Warren, W. C., and Hahn, M. W. (2014). Extensive error in the number of genes inferred from draft genome assemblies. PLoS Comput. Biol. 10:e1003998. doi: 10.1371/journal.pcbi.1003998

Dohm, J. C., Lottaz, C., Borodina, T., and Himmelbauer, H. (2008). Substanial biases in ultra-short read data sets from high-throughput dna sequencing. Nucleic Acids Res. 36:e105. doi: 10.1093/nar/gkn425

Drosophila 12 Genomes Consortium. (2007). Evolution of genes and genomes on the Drosophila phylogeny. Nature 450, 203-218. doi: 10.1038/nature 06341

Earl, D., Bradnam, K., St John, J., Darling, A., Lin, D., Fass, J., et al. (2011). Assemblathon 1: a competitive assessment of de novo short read assembly methods. Genome Res. 21, 2224-2241. doi: 10.1101/gr.126599.111

Eid, J., Fehr, A., Gray, J., Luong, K., Lyle, J., Otto, G., et al. (2008). Real-time DNA sequencing from single polymerase molecules. Science 323, 133-138. doi: $10.1126 /$ science. 1162986

Ekblom, R., and Wolf, J. B. W. (2014). A field guide to whole-genome sequencing, assembly and annotation, Evol. Appl. 7, 1026-1042. doi: 10.1111/eva.12178

Elshire, R. J., Glaubitz, J. C., Sun, Q., Poland, J. A., Kawamoto, K., Buckler, E. S., et al. (2011). A robust, simple genotyping-by-sequencing (GBS) approach for high diversity species. PLOS ONE 6:e19379. doi: 10.1371/journal.pone. 0019379

Gnerre, S., MacCallum, I., Przybylski, D., Ribeiro, F. J., Burton, J. N., Walker, B. J., et al. (2011). High-quality draft assemblies of mammalian genomes from massively parallel sequence data. Proc. Natl. Acad. Sci. U.S.A. 108, 1513-1518. doi: $10.1073 /$ pnas. 1017351108

Green, P. (1997). $2 \times$ genomes-Does depth matter? Genome Res. 7:410.

Hahn, M. W., Zhang, S. V., and Moyle, L. C. (2014). Sequencing, assembling, and correcting draft genomes using recombinant populations. G3 (Bethesda) 4, 669-679. doi: 10.1534/g3.114.010264

Heffelfinger, C., Fragoso, C. A., Moreno, M. A., Overton, J. D., Mottinger, J. P., Zhao, H., et al. (2014). Flexible and scalable genotyping-by-sequencing strategies for population studies. BMC Genomics 15:979. doi: 10.1186/14712164-15-979

Hunt, M., Kikuchi, T., Sanders, M., Newbold, C., Berriman, M., and Otto, T. (2013). REAPR: a universal tool for genome assembly evaluation. Genome Biol. 14:R47. doi: 10.1186/gb-2013-14-5-r47

Jansen, J., de Jong, A. G., and van Ooijen, J. W. (2001). Constructing dense genetic linkage maps. Theor. Appl. Genet. 102, 1113-1122. doi: 10.1007/s0012200 00489

Kaplan, N., and Dekker, J. (2013). High-throughput genome scaffolding from in vivo DNA interaction frequency. Nat. Biotechnol. 31, 1143-1147. doi: $10.1038 /$ nbt. 2768

Kawakami, T., Smeds, L., Backstrom, N., Husby, A., Qvarnstrom, A., Mugal, C. F., et al. (2014). A high-density linkage map enables a second-generation 
collared flycatcher assembly and reveals the patterns of avian recombination rate variation and chromosomal evolution. Mol. Ecol. 23, 4035-4058. doi: $10.1111 /$ mec. 12810

Kim, U., Shizuya, H., De Jong, P. J., Birren, B., and Simon, M. I. (1992). Stable propagation of cosmid-sized human DNA inserts in an F-factor based vector. Nucleic Acids Res. 20, 1083-1085.

Koren, S., and Phillippy, A. M. (2015). One chromosome, one contig: complete microbial genomes from long-read sequencing and assembly. Curr. Opin. Microbiol. 23, 110-120. doi: 10.1016/j.mib.2014.11.014

Koren, S., Harhay, G. P., Smith, T. P., Bono, J. L., Harhay, D. M., McVey, S. D., et al. (2013). Reducing assembly complexity of microbial genomes with single-molecule sequencing. Genome Biol. 14:R101. doi: 10.1186/gb-2013-149-r101

Kozarewa, I., Ning, Z., Quail, M. A., Sanders, M. J., and Berriman, M. (2009). Amplification-free Illumina sequencing-library preparation facilitates improved mapping and assembly of (G+C)-biased genomes. Nat. Methods 6, 291-295. doi: 10.1038/nmeth.1311

Lander, E. S., and Botstein, D. (1989). Mapping mendelian factors underlying quantitative traits using RFLP linkage maps. Genetics 121, 185-199.

Landolin, J., Chin, J., Kim, K., Yu, C., Fisher, W. W., Wan, K. H., et al. (2014). Initial De novo assemblies of the $D$. melanogaster genome using long-read PacBio sequencing. doi: 10.6084/m9.figshare.976097

Levy-Sakin, M., and Ebenstein, Y. (2013). Beyond sequencing: optical mapping of DNA in the age of nanotechnology and nanoscopy. Curr. Opin. Biotechnol. 24, 690-698. doi: 10.1016/j.copbio.2013.01.009

Lewin, H. A., Larkin, D. M., Pontius, J., and O'Brien, S. J. (2009). Every genome sequence needs a good map. Genome Res. 19, 1925-1928. doi: 10.1101/gr.094557.109

Lieberman-Aiden, E., van Berkum, N. L., Williams, L., Imakaev, M., Ragoczy, T., Telling, A., et al. (2009). Comprehensive mapping of long-range interactions reveals folding principles of the human genome. Science 326, 289-293. doi: $10.1126 /$ science. 1181369

Liu, L., Li, Y., Li, S., Hu, N., He, Y., Pong, R., et al. (2012). Comparison of next-generation sequencing systems. J. Biomed. Biotechnol. 2012, 1-11. doi: $10.1155 / 2012 / 373945$

Liu, D., Ma, C., Hong, W., Huang, L., Liu, M., Liu, H., et al. (2014). Construction and analysis of high-density linkage map using highthroughput sequencing data. PLoS ONE 9:e98855. doi: 10.1371/journal.pone. 0098855

Margarido, G. R. A., Souza, A. P., and Garcia, A. A. F. (2007). Onemap: software for genetic mapping in outcrossing species. Hereditas 144, 78-79. doi: 10.1111/j.2007.0018-0661.02000.x

Mascher, M., and Stein, N. (2014). Genetic anchoring of whole-genome shotgun assemblies. Front. Genet. 5:208. doi: 10.3389/fgene.2014.00208

Mascher, M., Muehlbauer, G. J., Rokhsar, D. S., Chapman, J., Schmutz, J., Barry, K., et al. (2013). Anchoring and ordering NGS contig assemblies by population sequencing (POPSEQ). Plant J. 76, 718-727. doi: 10.1111/tpj. 12319

McCoy, R. C., Taylor, R. W., Blauwkamp, T. A., Kelley, J. L., Kertesz, M., Pushkarev, D., et al. (2014). Illumina TruSeq synthetic long-reads empower de novo assembly and resolve complex, highly-repetitive transposable elements. PLoS ONE 9:e106689. doi: 10.1371/journal.pone.0106689

Mollinari, M., Margarido, G. R. A., Vencovsky, R., and Garcia, A. A. F. (2009). Evaluation of algorithms used to order markers on genetic maps. Heredity 103, 494-502. doi: 10.1038/hdy.2009.96

Myers, E. W., Sutton, G. G., Delcher, A. L., Dew, I. M., Fasulo, D. P., Flanigan, M. J., et al. (2000). A whole-genome assembly of Drosophila. Science 287, 2196-2204. doi: $10.1126 /$ science.287.5461.2196

Nossa, C. W., Havlak, P., Yue, J., Lv, J., Vincent, K. Y., Brockmann, H. J., et al. (2014). Joint assembly and genetic mapping of the atlantic horseshoe crab genome reveals ancient whole genome duplication. Gigascience 3:9. doi: 10.1186/2047-217X-3-9

O'Connor, M., Peifer, M., and Bender, W. (1989). Construction of large DNA segments in Escherichia coli. Science 4910, 1307-1312.

Petterson, E., Lundeberg, J., and Ahmadian, A. (2009). Generations of sequencing technologies. Genomics 93, 105-111. doi: 10.1016/j.ygeno.2008. 10.003
Pevzner, P. A., Tang, H., and Waterman, M. S. (2001). An Eulerian path approach to DNA fragment assembly. Proc. Natl. Acad. Sci. U.S.A. 98, 9748-9753. doi: $10.1073 /$ pnas. 171285098

Pop, M., and Salzberg, S. L. (2008). Bioinformatics challenges of new sequencing technology. Trends Genet. 24, 142-149. doi: 10.1016/j.tig.2007.12.006

Price, J. C., Udall, J. A., Bodily, P. M., Ward, J. A., Schatz, M. C., Page, J. T., et al. (2012). "De novo identification of "heterotigs" towards accurate and in-phase assemby of complex plant genomes," in Proceedings of The 2012 International Conference on Bioinformatics and Computational Biology (BIOCOMP12) (Las Vegas, NV).

Putnam, N. H., O'Connell, B., Stites, J. C., Rice, B. J., Fields, A., Hartley, P. D., et al. (2015). Chromsome-scale shotgun assembly using an in vitro method for long-range linkage. Available online at: http://arxiv.org/abs/1502.05331

Quail, M. A., Smith, M., Coupland, P., Otto, T. D., Harris, S. R., Connor, T. R., et al. (2012). A tale of three next generation sequencing platforms: comparison of Ion torrent, pacific biosciences and illumina miseq sequencers. BMC Genomics 13:341. doi: 10.1186/1471-2164-13-341

Quick, J., Quinlan, A., and Loman, N. (2014). A reference bacterial genome dataset generated on the MinION(TM) portable single-molecule nanopore sequencer. Gigascience 3, 22. doi: 10.1101/009613

Rastas, P., Paulin, L., Hanski, I., Lehtonen, R., and Auvinen, P. (2013). Lep-MAP: fast and accurate linkage map construction for large SNP datasets. Bioinformatics 29, 3128-3134. doi: 10.1093/bioinformatics/ btt563

Sanger, F., and Coulson, A. R. (1975). A rapid method for determining sequences in DNA by primed synthesis with DNA polymerase. J. Mol. Biol. 94, 441-448.

Sanger, F., Nicklen, S., and Couson, A. R. (1977). DNA sequencing with chainterminating inhibitors. Proc. Natl. Acad. Sci. U.S.A. 74, 5463-5467.

Schneider, G. F., and Dekker, C. (2012). DNA sequencing with nanopores. Nat. Biotechnol. 30, 326-328. doi: 10.1038/nbt.2181

Schwartz, D. C., Li, X., Hernandez, L., Ramnarain, S. P., Huff, E. J., and Wang, Y. K. (1993). Ordered restriction maps of Saccharomyces cerevisiae chromosomes constructed by optical mapping. Science 262, 110-114.

Semagn, K., Bjørnstad, A., and Ndjiondjop, M. J. (2006). Principles, requirements and prospects of genetic mapping in plants. Afr. J. Biotechnol. 5, 2569-2587.

Shizuy, H., Birren, B., Kim, U., Mancino, V., Slepak, T., Tachiiri, Y., et al. (1992). Cloning and stable maintenance of 300-kilobase-pair fragments of human DNA in Escherichia coli using an F-factor-based vector. Proc. Natl. Acad. Sci. U.S.A. $89,8794-8797$

Stam, P. (1993). Construction of integrated genetic linkage maps by means of a new computer package: joinmap. Plant J. 3, 739-744.

Strnadova, V., Buluc, A., Chapman, J., Gilbert, J. R., Gonzalez, J., Jegelka, S., et al. (2014). "Efficient and accurate clustering for large-scale genetic mapping," in 2014 IEEE International Conference on Bioinformatics and Biomedicine (Belfast: IEEE), 3-10. doi: 10.1109/BIBM.2014.6999119

Sturtevant, A. H. (1913a). The linear arrangement of six sex-linked factors in Drosophila, as shown by their mode of association. J. Exp. Zool. 14 43-59.

Sturtevant, A. H. (1913b). A third group of linked genes in Drosophila ampelophila. Science 37, 990-992.

Suomalainen, E., Cook, L. M., and Turner, J. R. G. (1973). Achiasmatic oogenesis in the heliconiine butterflies. Hereditas 74, 302-304.

The International Wheat Genome Sequencing Consortium. (2014). A chromsomebased draft sequence of the hexaploid bread wheat (Triticum aestivum) genome. Science 345, 1-11. doi: 10.1126/science. 1251788

van Oers, K., Santure, A. W., De Cauwer, I., van Bers, N. E. M., Crooijmans, R. P. M. A., Shelder, B. C., et al. (2014). Replicated high-density genetic maps of two great tit populations reveal fine-scale genomic departures from sex-equal recombination rates. Heredity 112, 307-316. doi: 10.1038/hdy. 2013.107

van Ooijen, J. W. (2011). Multipoint maximum likelihood mapping in a full-sib family of an outbreeding species. Genet. Res. 93, 343-349. doi: $10.1017 /$ S0016672311000279

Weber, J., and Myers, H. (1997). Human whole-genome shotgun sequencing. Genome Res. 7:401. 
Wu, Y., Bhat, P. R., Close, T. J., and Lonardi, S. (2008). Efficient and accurate construction of genetic linkage maps from the minimum spanning tree of a graph. PLoS Genet. 4:e1000212. doi: 10.1371/journal.pgen. 1000212

Wu, J., Jenkins, J. N., McCarty, J. C., and Lou, X. (2011). Comparisons of four approximation algorithms for large-scale linkage map construction. Theor. Appl. Genet. 123, 649-655. doi: 10.1007/s00122-0111614-8

Xie, T., Zheng, J. F., Liu, S., Peng, C., Zhou, Y. M., Yang, Q. Y., et al. (2015). De novo plant genome assembly based on chromatin interactions: a case study of Arabidopsis thaliana. Mol. Plant 8, 489-492. doi: 10.1016/j.molp.2014.12.015

Xu, X., Pan, S., Cheng, S., Zhang, B., Mu, D., Ni, P., et al. (2011). Genome sequence and analysis of the tuber crop potato Nature 475, 189-195. doi: $10.1038 /$ nature 10158
Zerbino, D. R., and Birney, E. (2010). Velvet: algorithms for de novo short read assembly using de Bruijn graphs. Genome Res. 18, 821-829. doi: 10.1101/gr.074492.107

Conflict of Interest Statement: The author declares that the research was conducted in the absence of any commercial or financial relationships that could be construed as a potential conflict of interest.

Copyright $(2015$ Fierst. This is an open-access article distributed under the terms of the Creative Commons Attribution License (CC BY). The use, distribution or reproduction in other forums is permitted, provided the original author(s) or licensor are credited and that the original publication in this journal is cited, in accordance with accepted academic practice. No use, distribution or reproduction is permitted which does not comply with these terms. 